\begin{tabular}{cl}
$p$ & \multicolumn{1}{c}{ Factors of $M_{p}$} \\
8167 & 76835137 \\
8171 & 9412993 \\
8209 & 14759783 \\
8221 & $9667897 \cdot 18480809$ \\
8269 & 19630607 \\
8273 & $28062017 \cdot 62014409$ \\
8287 & 36877151 \\
8377 & $134033 \cdot 787439 \cdot 2596871$ \\
8429 & $455167 \cdot 927191$ \\
8467 & 6655063 \\
8539 & 13662401 \\
8563 & 32402393 \\
8573 & 12345121 \\
8623 & 80504329 \\
8699 & 43790767 \\
8737 & 6640121 \\
8741 & 5926399 \\
8849 & 52368383 \\
8933 & 36232249 \\
8969 & 13345873 \\
9029 & 25913231
\end{tabular}

\begin{tabular}{rl}
$p$ & \multicolumn{1}{c}{ Factors of $M_{p}$} \\
9049 & $28721527 \cdot 28938703$ \\
9059 & 30293297 \\
9109 & 49625833 \\
9127 & 8707159 \\
9137 & 2704553 \\
9161 & 86901247 \\
9199 & 53354201 \\
9221 & 91841161 \\
9283 & $29352847 \cdot 34031479 \cdot 41532143$ \\
9337 & $2838449 \cdot 2405633$ \\
9403 & 5735831 \\
9479 & 48532481 \\
9601 & $3513967 \cdot 16974569 \cdot 17256487$ \\
9643 & 12362327 \\
9743 & 34626623 \\
9817 & 20556799 \\
9829 & 14075129 \\
9851 & 3723679 \\
9859 & 1656313 \\
9883 & 10436449 \\
9973 & $7419913 \cdot 10591327 \cdot 19367567$
\end{tabular}

\title{
Certain Properties of Pyramidal and Figurate Numbers
}

\author{
By M. Wunderlich
}

It is well known that despite some extensive computation [1], the only two known solutions to the Diophantine equation

$$
a^{3}+b^{3}+c^{3}=3
$$

are $a=b=c=1$, and $a=b=4, c=-5$. Professor Aubrey Kempner noted at a number theory seminar at the University of Colorado that these solutions also satisfy the equation

$$
a^{3}+b^{3}+c^{3}=a+b+c .
$$

Therefore, it is of interest whether or not (2) has solutions other than these two and if so, how many. Since there are so few solutions known to (1), it seemed reasonable to conjecture that there would be only finitely many solutions to (2).

If we change the sign of the third variable and divide through by six, we see that (2) is equivalent to

$$
\frac{a^{3}-a}{6}+\frac{b^{3}-b}{6}=\frac{c^{3}-c}{6}
$$

or

$$
\frac{(a-1)(a)(a+1)}{6}+\frac{(b-1)(b)(b+1)}{6}=\frac{(c-1)(c)(c+1)}{6} .
$$

Received March 13, 1962. The research for this paper was supported in part by the National Science Foundation. 
Numbers of the form

$$
\frac{(a-1)(a)(a+1)}{6}, \quad a \geqq 2
$$

had been studied by the ancients and had been given the name "Pyramidal" because of a curious geometric property which they possess [2]. It is also clear from (3) that we can define the pyramidal numbers, $P_{n}$, as follows:

$$
P_{n}=\left(\begin{array}{c}
n+2 \\
3
\end{array}\right), \quad n \geqq 1 .
$$

In view of the binomial theorem, they are the numbers in the fourth diagonal row of Pascal's triangle, a fact which makes the numerical computation of these numbers very easy.

Therefore, the conjecture mentioned concerning (2) can be restated as follows: There is only a finite number of solutions to

$$
P_{x}+P_{y}=P_{z} \text {. }
$$

The purpose of this paper is to indicate how the reasonableness of this conjecture was tested and how an examination of a table of solutions of (4) led to some interesting theoretical results. In particular, S. Chowla was able to prove that there are infinitely many solutions to (4) and hence to (2) [3]. Also, S. Segal could prove that the only solution to

$$
2 P_{x}=P_{y}
$$

is where $x=3$ and $y=4$ [4].

The computation of the first 88 solutions of (4) was done with the help of V. Keiser on the C.D.C. 1604 digital computer at the National Bureau of Standards laboratories in Boulder, Colorado. The method used was systematically to determine for every pair of pyramidal numbers $P_{n}, P_{m}, m<13,000, n<m$, whether or not $P_{m}-P_{n}$ was again pyramidal. If we let $M=P_{m}-P_{n}$ it is necessary to determine whether

$$
M=P_{v}, \quad v=1,2, \cdots n-1 .
$$

To do this, the following, "hunting" procedure was employed: All the numbers $P_{1}, P_{2}, \cdots P_{n}$ were stored in the machine in ascending order of magnitude. $M$ was first compared with $P_{[n / 2]}$. ([n] as usual indicates the integral part of $n$.) If $M=$ $P_{[n / 2]}$, a solution to (4) was found. If $M<P_{[n / 2]}$, a solution can only exist for $v=1,2, \cdots,[n / 2]-1$. If $M>P_{[n / 2]}$, a solution can only exist for $v=[n / 2]+$ $1,[n / 2]+2, \cdots, n$. In either case the number of values which $v$ can assume is roughly halved. If this procedure is repeated $[\log m / \log 2]+1$ times, any solution if it exists will be found. For example, if $m=15,000$, the process need only be repeated 14 times.

Table 1 lists the first 88 solutions found using this program. It required approximately 6 hours of machine time.

It is interesting to note that although $\mathrm{S}$. Chowla proved that there exist infinitely many solutions to (4), he by no means justified the great number of solutions that were found. By imposing extra relations on (2) he was able to reduce it to a Pellian equation which has infinitely many solutions that also satisfy (2). Therefore, he 
TABLE 1

\begin{tabular}{|c|c|c|c|c|c|}
\hline$x$ & $y$ & $z$ & $x$ & $y$ & $z$ \\
\hline 3 & 3 & 4 & 1351 & 1478 & 1786 \\
\hline 8 & 14 & 15 & 798 & 1818 & 1868 \\
\hline 20 & 54 & 55 & 438 & 2164 & 2170 \\
\hline 30 & 55 & 58 & 1146 & 2072 & 2183 \\
\hline 39 & 70 & 74 & 1139 & 2115 & 2220 \\
\hline 61 & 102 & 109 & 1609 & 1941 & 2256 \\
\hline 84 & 90 & 110 & 1105 & 2303 & 2385 \\
\hline 34 & 118 & 119 & 853 & 2417 & 2452 \\
\hline 48 & 138 & 140 & 1103 & 2514 & 2583 \\
\hline 119 & 154 & 175 & 1484 & 2584 & 2738 \\
\hline 187 & 201 & 245 & 1089 & 2773 & 2828 \\
\hline 100 & 290 & 294 & 834 & 2958 & 2980 \\
\hline 327 & 336 & 418 & 528 & 3138 & 3143 \\
\hline 149 & 429 & 435 & 1775 & 2954 & 3154 \\
\hline 252 & 424 & 452 & 1484 & 3094 & 3204 \\
\hline 248 & 450 & 474 & 2478 & 2726 & 3286 \\
\hline 362 & 415 & 492 & 2099 & 3211 & 3486 \\
\hline 219 & 515 & 528 & 729 & 3595 & 3605 \\
\hline 136 & 532 & 535 & 2200 & 3660 & 3908 \\
\hline 424 & 448 & 550 & 742 & 4415 & 4422 \\
\hline 314 & 527 & 562 & 2116 & 4580 & 4726 \\
\hline 434 & 495 & 588 & 2948 & 4408 & 4810 \\
\hline 399 & 588 & 644 & 3138 & 4630 & 5068 \\
\hline 324 & 663 & 688 & 2912 & 4838 & 5167 \\
\hline 272 & 688 & 702 & 868 & 6034 & 6040 \\
\hline 304 & 695 & 714 & 2252 & 6390 & 6482 \\
\hline 349 & 713 & 740 & 5338 & 5608 & 6900 \\
\hline 532 & 643 & 747 & 3570 & 7154 & 7439 \\
\hline 424 & 705 & 753 & 1271 & 7554 & 7566 \\
\hline 378 & 790 & 818 & 6152 & 6586 & 8034 \\
\hline 608 & 754 & 868 & 1160 & 8070 & 8078 \\
\hline 230 & 903 & 908 & 5300 & 7284 & 8120 \\
\hline 489 & 869 & 918 & 5630 & 7105 & 8129 \\
\hline 775 & 950 & 1098 & 6340 & 6788 & 8280 \\
\hline 703 & 1064 & 1158 & 4115 & 8034 & 8379 \\
\hline 878 & 1044 & 1220 & 4015 & 8910 & 9174 \\
\hline 968 & 1001 & 1241 & 7104 & 7847 & 9442 \\
\hline 922 & 1286 & 1428 & 7062 & 8094 & 9592 \\
\hline 290 & 1430 & 1434 & 2951 & 10184 & 10266 \\
\hline 367 & 1436 & 1444 & 1328 & 10568 & 10575 \\
\hline 855 & 1343 & 1450 & 7842 & 10168 & 11532 \\
\hline 504 & 1629 & 1645 & 7294 & 10618 & 11660 \\
\hline 897 & 1621 & 1708 & 8274 & 10149 & 11725 \\
\hline 750 & 1690 & 1738 & 9050 & 11100 & 12824 \\
\hline
\end{tabular}

found infinitely many solutions of a very special type, none of which, incidentally, appear in the table.* Two unsolved problems are to find a parametric representation

* (Added in publication) In the March 1961 issue of Elemente Der Mathematik, W. Sierpinski has also shown that there are infinitely many such solutions. His proof, however, does yield two of the solutions in the table, namely $x=8, y=14, z=15$ and $x=2912$, $y=4838, z=5167$. 
which will give all the solutions to (2) and to find an asymptotic density function analogous to the prime number theorem. This latter problem can be more explicitly stated as follows: Let $\phi(x)$ represent the number of integers $n \leqq x$ such that there exist positive integers $a, b$ such that

$$
P_{a}+P_{b}=P_{n} \text {. }
$$

Does there exist a continuous function $g(x)$ such that $\phi(x) \sim g(x)$ ? That is,

$$
\lim _{x \rightarrow \infty} \frac{\phi(x)}{g(x)}=1 \text {. }
$$

The concept of pyramidal number can be generalized by defining the rth figurate number of order $n$ to be the binomial coefficient

$$
f_{n, r}=\left(\begin{array}{c}
r+n-1 \\
n
\end{array}\right) \text {. }
$$

In this notation the pyramidal number $P_{x}$ is $f_{3, x}$. Work is now in progress to compute possible solutions to

$$
f_{n, x}+f_{n, y}=f_{n, z}
$$

where $n=4,5$, and 6 . One might be led to believe that there are only finitely many. solutions to (5) for $n=4$ for the following reason: Whereas for $n=3$ the equation was reduced to a Pellian equation, if $n=4$ a similar reduction may result in a set of cubic Diophantine equations to which the Roth theorem may apply. If the reduction could be effected without imposing any further restrictive relations the conjecture would be proved. Preliminary results show that the only two solutions to (5) for $n=4$ and $z<5264$ are $x=4, y=4, z=5$; and $x=129, y=187$, $z=197$. It is interesting to note that Paul Erdos had once conjectured in a letter to Mr. Chowla that the only solution of

$$
2 f_{n, x}=f_{n, y}
$$

is $x=n$ and $y=n+1$. As we have seen, $\mathrm{S}$. Segal has affirmatively confirmed this conjecture for $n=3$.

It was further noted upon examining a decimal print-out of the first 25,000 pyramidal numbers that the last digit repeated itself in a cycle of 20 , i.e.,

$$
\text { If } x \equiv y \quad(\bmod 20) \text {, then } f_{3, x} \equiv f_{3, y} \quad(\bmod 10) .
$$

This observation led to the following generalized result:

Theorem. If $k=p_{1}^{\gamma_{1}}{p_{2}}^{\gamma_{2}} \cdots p_{q}^{\gamma_{q}}$, and $n$ is a positive integer then for $j=1$, $2, \cdots q$ let

$$
\beta_{j}=\left[\frac{n}{p_{j}}\right]+\left[\frac{n}{p_{j}^{2}}\right]+\cdots+\left[\frac{n}{p_{j}^{\alpha_{j}}}\right]
$$

where $p_{j}^{\alpha_{j}}$ is the largest power of $p_{j} \leqq n$. i.e. $\alpha_{j}=\left[\log n / \log p_{j}\right]$. Finally let $t=p_{1}^{\beta_{1}}$ $p_{2}^{\beta_{2}} \cdots p_{q}^{\beta_{q}}$. If $x$ and $y$ are positive integers such that $x \equiv y(\bmod t k)$, then

$$
f_{n, x} \equiv f_{n, y} \quad(\bmod k)
$$

(Note that (6) is a special case of the theorem where $n=3$ and $k=10$.) 
Proof. For $i=1,2, \cdots n,(x+n-i) \equiv(y+n-i) \quad(\bmod t k)$ so that $(x+n-1)(x+n-2) \cdots(x) \equiv(y+n-1)(y+n-2) \cdots(y) \quad(\bmod t k)$. Also from the definition of $t,(n !, t k)=t$ since $t$ is the product of highest powers of $p_{1}, p_{2}, \cdots p_{q}$ which are contained in $n$ !. So,

$$
f_{n, x}=\frac{(x+n-1) \cdots(x)}{n !} \equiv \frac{(y+n-1) \cdots(y)}{n !}=f_{n, y}(\bmod k)
$$

which proves the theorem.

It is not asserted in the theorem that $t k$ is the smallest period. In fact, easy examples show that in many cases a value for $t$ can be found which is strictly smaller than the one specified in the theorem. According to Mathematical Reviews (v. 20, 1959, Review no. 1653), the smallest period has evidently been found by S. Zabek [5] to be $t k$ where

$$
t={p_{1}}^{\alpha_{1}}{p_{2}}^{\alpha_{2}} \cdots p_{q}^{\alpha_{q}} \text {. }
$$

This information may be quite useful in numerically searching for solutions to (5) since these congruences limit the number of solutions that could possibly exist, thereby reducing the amount of machine time needed for the search.

The University of Colorado

Boulder, Colorado

1. J. C. P. Miller, \& M. F. C. Woollett, "Solutions of the Diophantine equation $x^{3}+$ $y^{3}+z^{3}=k$," London Math. Soc., v. 30, 1955, p. 101-110.

2. L. Dickson, History of the Theory of Numbers, v. 2, Chap. 1. (Reprint: Stechert, New York, 1934).

3. S. CHOwla and others, "The Diophantine equation $x^{3}+y^{3}+z^{3}=x+y+z$," submitted to Norske Vid. Selsk. Forh. Trondheim.

4. S. Segal, "A note on pyramidal numbers," American Math. Monthly, (in press).

5. Swiatomir Zabex, "Sur la periodicite modulo $m$ des suites de nombres $\left(\begin{array}{l}n \\ k\end{array}\right)$, , Ann. Univ. Mariae Curi-Sklodowska, Sect. A, 10 (1956), p. 37-47.

\section{Note on Osculatory Rational Interpolation $\dagger$}

\section{By Herbert E. Salzer}

Abstract. In $n$-point osculatory interpolation of order $r_{i}-1$ at points $x_{i}$, $i=1,2, \cdots, n$, by a rational expression $N(x) / D(x)$, where $N(x)$ and $D(x)$ are polynomials $\sum a_{j} x^{j}$ and $\sum b_{j} x^{j}$, we use the lemma that the system (1) $\left\{N\left(x_{i}\right) / D\left(x_{i}\right)\right\}^{(m)}=f^{(m)}\left(x_{i}\right), m=0,1, \cdots, r_{i}-1$, is equivalent to (2) $N^{(m)}\left(x_{i}\right)=$ $\left\{f\left(x_{i}\right) D\left(x_{i}\right)\right\}^{(m)}, m=0,1, \cdots, r_{i}-1, D\left(x_{i}\right) \neq 0$. This equivalence does not require $N(x)$ or $D(x)$ to be a polynomial or even a linear combination of given functions. The lemma implies that (1), superficially non-linear in $a_{j}$ and $b_{j}$, being the same as (2), is actually linear. For the $n$-point interpolation problem, the linear system, of order $\sum_{i=1}^{n} r_{i}$, which might be large, is replaceable by separate linear

Received April 20, 1961.

$\dagger$ Much of the material in the present note is contained in an entirely independent (still unpublished) study by Henry C. Thacher, Jr., who was kind enough to send the writer a copy of his preliminary draft. 